\title{
DESIDRATAÇÃO OSMÓTICA DE FILÉS DE PIRARUCU (Arapaima gigas)
}

\section{OSMOTIC DEHYDRATION OF FILLETS PIRARUCU (Arapaima gigas)}

\author{
Renan de Almeida Maciel ${ }^{1}$; Camila Maria Corrêa Viana²; Marilda Garcia Simões ${ }^{3}$; Mauro José Pantoja \\ Fontelles ${ }^{4}$ \\ ${ }^{1,2}$ Universidade do Estado do Pará - UEPA - Belém - Brasil \\ ${ }^{3}$ Universidade do Estado do Pará - UEPA - Belém - Brasil marildags@uol.com.br \\ ${ }^{4}$ Universidade do Estado do Pará - UEPA - Belém - Brasil
}

\begin{abstract}
Resumo
Tratamentos osmóticos são usados principalmente como um pré-tratamento, sendo introduzidos em alguns processos convencionais para melhorar a qualidade do produto final e reduzir custos de energia, ou mesmo para a formulação de novos produtos. O presente estudo otimizou, pela aplicação da metodologia de superfície de resposta, a desidratação osmótica de filés de pirarucu (Arapaima gigas). Um planejamento fatorial completo $2^{3}$ foi desenvolvido envolvendo as variáveis independentes temperatura, concentração de $\mathrm{NaCl}$ e tempo de imersão, e as variáveis dependentes perda de peso, perda de água e ganho de sólidos. A concentração de $\mathrm{NaCl}$ e a temperatura foram as variáveis que mais influenciaram na perda de peso e perda de água das amostras de filé de pirarucu desidratadas com $\mathrm{NaCl}$, apresentando um efeito positivo A temperatura e a interação tempo x temperatura apresentaram efeito significativo negativo, enquanto a concentração de $\mathrm{NaCl}$ e a interação concentração de $\mathrm{NaCl} x$ tempo, apresentaram efeito significativo positivo para a resposta ganho de sólidos. O tempo de desidratação osmótica não exerceu influência nas respostas envolvidas no estudo. A sobreposição das superfícies de respostas da perda de água e ganho de sólidos indicou que a temperatura de $49{ }^{\circ} \mathrm{C}$ e a concentração de $28 \%$ mais viável no sentido de se obter maior perda de água e o menor ganho de sólidos.
\end{abstract}

Palavras-chave: desidratação osmótica; pirarucu; planejamento experimental.

\section{Introdução}

A operação unitária conhecida como desidratação osmótica, também denominada de impregnação por imersão, é um processo que consiste na imersão de alimentos, inteiro ou em pedaços, em soluções aquosas hipertônicas de alta pressão osmótica (por exemplo: sais, açúcar, sorbitol, glicerol etc.), o que provoca a remoção da água presente no alimento, de forma a reduzir a sua disponibilidade para deterioração química, microbiológica e sensorial (RAOULT - WACK et al., 1989; TORREGGIANI, 1993). 
Durante a desidratação osmótica, pelo menos dois fluxos ocorrem simultaneamente e em contra corrente: um destes fluxos é o de saída da água do produto em direção à solução; o outro é a migração de solutos da solução para o alimento, ambos através da membrana celular. Um terceiro fluxo, que envolve a perda de alguns sólidos naturais como açúcares, minerais, ácidos orgânicos e vitaminas, também ocorre durante esse processo, e mesmo sendo insignificante aos dois fluxos principais, é de fundamental importância para as qualidades sensoriais de aroma, cor e textura, assim como para as qualidades nutricionais, minerais e das vitaminas do produto final (RAOULT WACK et al., 1994).

Este processo que vem sendo usado como pré-tratamento antes da secagem e refrigeração de alimentos, representa um meio para diminuir a umidade e melhorar a qualidade do produto final, além de reduzir custos de energia, ou mesmo para a formulação de novos produtos. Para o caso do pescado, a imersão em salmouras concentradas é uma técnica amplamente usada, cujo processo origina produtos com boas condições de armazenamento, com qualidade semelhante à matériaprima e, na maioria das vezes, com melhor coloração, sabor e aroma, quando comparados aos produtos elaborados com tecnologias mais caras e mais sofisticadas (SERENO et al., 2001; REYES et al., 2005; GOMES et al., 2007).

O pirarucu (Arapaima gigas), em razão de seu grande porte, é o peixe mais famoso e emblemático da ictiofauna amazônica, representando papel histórico na pesca e no desenvolvimento sócio-econômico da região. É um peixe endêmico, exclusivo da região, sendo encontrado nas águas calmas do rio Amazonas e afluentes, como, também, nas vastas áreas alagáveis de várzea e igapó, ligados a ele (BARD e IMIRIBA, 1986; IMBIRIBA, 2001). É a espécie mais promissora para o desenvolvimento da piscicultura em regime intensivo na região Amazônica, pois possui alta taxa de crescimento, podendo alcançar de 7 a $10 \mathrm{~kg}$ no primeiro ano de vida (IMBIRIBA, 2001; PEREIRAFILHO et al., 2002; ONO et al., 2004).

Sua carne, extremamente saborosa, é apresentada ao consumidor principalmente na forma seca e salgada, em mantas, sendo dessa forma conhecido como o "bacalhau brasileiro". Este processo artesanal de salga, que possibilita um ganho de sal tecidual, ocasiona mudanças em suas características sensoriais originais, a qual é ocasionada pela desnaturação das proteínas e pela oxidação de lipídeos, fato este que requer a busca de fórmulas ideais de modo a preservar as características originais do produto (OGAWA, 1999; ONO et al., 2004).

Assim, o objetivo do presente estudo foi, pela aplicação do método de análise de superfície de resposta, obter a melhor condição de processamento para a desidratação osmótica de filés de pirarucu. 


\section{Metodologia}

Preparo das Amostras

O presente estudo utilizou filés de pirarucu fresco, adquiridos no mercado Atlântida Pescado, localizado no município de Belém - PA. As amostras foram acondicionadas em isopor com gelo e transportadas para o Laboratório de Alimentos - UEPA, onde foram acondicionados em freezer a $-18{ }^{\circ} \mathrm{C}$. Para as análises microbiológicas e físico-químicas dos filés in natura, foram utilizadas porções homogeneizadas da região dorsal do peixe, tendo em vista a possível diferença de composição química do músculo na região distal e proximal. Para desidratação osmótica, os filés foram cortados com auxílio de faca inox, em cubos de aresta igual a $1 \mathrm{~cm}$.

\section{Análises microbiológicas}

Como as amostras foram manipuladas antes de chegar ao Laboratório de Alimentos, foi realizada a análise de coliformes termotolerantes, de acordo com a Portaria 451 de 19/09/1997 do Ministério da Saúde, para verificar a sanidade do lote recebido (BRASIL, 1997). Para a avaliação da qualidade microbiológica da matéria-prima, realizaram-se a pesquisa de Salmonella e a contagem de Staphylococcus aureus, de acordo com as determinações da RDC N ${ }^{\circ} 12$, de janeiro de 2001 (BRASIL, 2001).

\section{Análises físico-químicas}

Para caracterização físico-química foram realizadas as análises de umidade, cinzas, proteínas e lipídeos, de acordo com AOAC (1997). A umidade foi determinada por método gravimétrico por secagem em estufa a $105{ }^{\circ} \mathrm{C}$, até peso constante. As cinzas foram determinadas por método gravimétrico, por calcinação, através da incineração da matéria orgânica, em forno mufla, a $550{ }^{\circ} \mathrm{C}$. A proteína foi calculada mediante a determinação de nitrogênio total pelo método de micro Kjeldahl. O lipídeo foi determinado por extração intermitente da fração lipídica com solvente orgânico (éter de petróleo).

\section{Desidratação osmótica}

A matéria-prima, depois de cortada em cubos de $1 \mathrm{~cm}$ de aresta, foi pesada e colocada em recipientes de vidro de $300 \mathrm{~mL}$, contendo solução de $\mathrm{NaCl}$. Foi utilizada uma relação volume da amostra/volume da solução de 1:30, de modo a garantir que a concentração da solução osmótica 
permanecesse constante ao longo do processo. Os recipientes foram, então, colocados em uma incubadora refrigerada (Shaker), aquecidos à temperatura do processo, com agitação constante de 80 rpm, garantindo uma movimentação satisfatória da amostra dentro da solução, para que a mesma não sofresse impacto com as paredes do becker. Os experimentos foram realizados em duplicata.

Após tempos pré-determinados pelo planejamento experimental, as amostras foram retiradas da incubadora, lavadas com água destilada para retirar o excesso da solução desidratante, secas em papel absorvente e novamente pesadas.

\section{Tratamento estatístico dos dados}

Os ensaios experimentais foram realizados de acordo com um delineamento composto central rotacional, em soluções binária de água $+\mathrm{NaCl}$. Foi determinado um planejamento fatorial completo $2^{3}$ de $1^{\mathrm{a}}$ ordem, envolvendo as variáveis independentes (preditoras): temperatura $(\mathrm{T})$, concentração de $\mathrm{NaCl}(\mathrm{C})$ e tempo de imersão (t), as quais foram avaliadas em dois níveis $(-1$ e +1$)$ com três repetições no ponto central (0), totalizando 11 experimentos. As variáveis dependentes (respostas) do planejamento foram: a perda de peso (PP), a perda de água (PA), o ganho de sólidos (GS).

Através do presente estudo foi possível obter modelos estatísticos capazes de predizer o comportamento das variáveis dependentes em função das variáveis independentes, na faixa adotada para a análise. Assume-se existir uma função matemática Y para cada resposta, em função das três variáveis independentes, conforme a equação abaixo.

$$
\mathrm{Y}=f(T, C, t)=\beta_{o}+\beta_{1} T+\beta_{11} T^{2}+\beta_{2} C+\beta_{22} \mathrm{C}^{2}+\beta_{3} \mathrm{t}+\beta_{33} \mathrm{t}^{2}+\beta_{12} T C+\beta_{13} T t+\beta_{23} C t
$$

Os resultados foram compilados em tabelas do programa Microsoft Office Excel (2003). Posteriormente, foram submetidos a tratamentos estatísticos utilizando-se o Programa Statistic for Windows versão 5.0 (1995), para determinar o efeito das variáveis independentes sobre as variáveis respostas. Foram verificadas através do erro puro e do SS residual quais variáveis apresentavam efeito estatisticamente significativo.

Após a análise estatística dos coeficientes, uma análise de variância (ANOVA), que consiste na avaliação do coeficiente de determinação $\left(\mathrm{R}^{2}\right)$ e do teste $\mathrm{F}$, verificou se o modelo apresenta um grau de ajuste adequado aos dados experimentais. As análises estatísticas foram processadas para comparação com nível alfa, o qual foi estabelecido em $5 \%(\mathrm{P} \leq 0,05)$. 
A melhor condição de processamento de desidratação osmótica foi determinada utilizando Metodologia de Superfície de Resposta (RSM), a qual é realizada pela sobreposição dos gráficos de PA e SG, de modo a maximizar a perda de água e minimizar o ganho de sólidos.

\section{Resultados e Discussões}

\section{Análise microbiológica da matéria-prima}

A Tabela 1 apresenta os resultados das análises microbiológicas do filé de pirarucu in natura.

Tabela 1 - Análise microbiológica do filé de pirarucu in natura

\begin{tabular}{c|c|c}
\hline Análises & Pirarucu in natura & Legislação (BRASIL, 2001) \\
\hline Salmonella spp. & Ausência em $25 \mathrm{~g}$ & Ausência em $25 \mathrm{~g}$ \\
Staphylococcus aureus & Coagulase Negativa & Máx. $10^{3} / \mathrm{g}$ \\
Coliformes $45^{\circ} \mathrm{C}$ & $9,2 \mathrm{NMP} * / \mathrm{g}$ & $\mathrm{Máx.} 10^{2} / \mathrm{g}$ \\
\hline *NMP: Número mais provável &
\end{tabular}

A ausência de Salmonella spp. e Staphylococcus aureus nas amostras analisadas indica que condições higiênico-sanitárias adequadas e satisfatórias foram adotadas, desde a captura do pescado até a chegada do mesmo ao Laboratório, permitindo, assim, a sua utilização no processo da desidratação osmótica.

\section{Análise físico-química}

Os resultados da caracterização físico-química do lote de filés de pirarucu, utilizado no estudo, encontram-se na Tabela 2.

Tabela 2 - Análise centesimal do filé de pirarucu in natura

\begin{tabular}{l|c}
\hline \multicolumn{1}{c|}{ Análises (n=3) } & Média \pm Desvio padrão \\
\hline Umidade & $79,58 \pm 0,92$ \\
Proteínas & $17,01 \pm 0,51$ \\
Lipídeos & $0,86 \pm 0,03$ \\
Cinzas & $0,65 \pm 0,04$ \\
Carboidratos & $1,82 \pm 0,26$ \\
Valore energético (Kcal/100g) & $83,03 \pm 1,05$ \\
\hline
\end{tabular}


Os valores apresentados na Tabela 2 estão próximos aos encontrados por Carvalho (1998), que apresentou valores de umidade igual a $80,4 \%$, proteína próximo a $18,0 \%$, lipídeos igual a $0,4 \%$ e cinzas próximo a $0,7 \%$.

Segundo Oliveira (2006), o pirarucu apresenta uma grande variabilidade quanto à composição físico-química determinada entre a porção dorsal e a porção abdominal do filé. Este autor constatou que a porção abdominal apresenta maior quantidade de lipídeos (2,49\%) em relação à porção dorsal $(0,62 \%)$. No presente trabalho, o valor encontrado para lipídeos dos filés de pirarucu foi de $0,86 \%$, o que corresponde à região dorsal do pirarucu.

De acordo com Pigott e Tucker (1990), uma forma de classificar os peixes quanto ao teor de lipídeos, está baseada na seguinte relação: peixes com concentração de lipídeo menor que $2 \%$ são caracterizados como um pescado de baixo conteúdo de gordura; teor de lipídeo entre $2 \%$ e $5 \%$ caracteriza um pescado com moderado conteúdo de gordura e, peixes com teor maior que 5\% são considerados um pescado com alto conteúdo de gordura. Os resultados deste estudo classificam o dorso do pirarucu como um pescado com baixo teor de gordura.

\section{Desidratação osmótica de filés de pirarucu}

Os valores de perda de peso, perda de água e ganho de sólidos, obtidos experimentalmente no processo de desidratação osmótica do filé de pirarucu, seguindo a planilha do planejamento experimental completo $2^{3}$, com oito pontos fatoriais e três centrais, estão apresentados na Tabela 3 .

Tabela 3 - Resultados da PP, PA e SG obtidos experimentalmente através da desidratação osmótica de filé de pirarucu utilizando solução binária $(\mathrm{NaCl}+$ Água)

\begin{tabular}{|c|c|c|c|c|c|c|c|c|c|}
\hline & \multicolumn{3}{|c|}{ Codificado } & \multicolumn{3}{|c|}{ Real } & \multicolumn{3}{|c|}{ Respostas } \\
\hline & $\mathrm{T}\left({ }^{\circ} \mathrm{C}\right)$ & $\mathrm{C}(\%)$ & $\begin{array}{c}\mathrm{t} \\
\text { (h) }\end{array}$ & $\mathrm{T}\left({ }^{\circ} \mathrm{C}\right)$ & $\mathrm{C}(\%)$ & $\begin{array}{c}\mathrm{t} \\
\text { (h) }\end{array}$ & $\begin{array}{l}\mathrm{PP} \\
(\%)\end{array}$ & $\begin{array}{l}\text { PA } \\
(\%) \\
\end{array}$ & $\begin{array}{l}\text { GS } \\
(\%)\end{array}$ \\
\hline 1 & -1 & -1 & -1 & 30 & 23 & 90 & 6,4243 & 17,4335 & 11,0092 \\
\hline 2 & +1 & -1 & -1 & 50 & 23 & 90 & 12,7464 & 25,0726 & 12,3262 \\
\hline 3 & -1 & +1 & -1 & 30 & 29 & 90 & 13,4569 & 25,6688 & 12,2119 \\
\hline 4 & +1 & +1 & -1 & 50 & 29 & 90 & 24,2138 & 36,2745 & 12,0607 \\
\hline 5 & -1 & -1 & +1 & 30 & 23 & 150 & 8,9768 & 20,9322 & 11,9554 \\
\hline 6 & +1 & -1 & +1 & 50 & 23 & 150 & 19,0406 & 29,4204 & 10,3798 \\
\hline 7 & -1 & +1 & +1 & 30 & 29 & 150 & 18,9118 & 31,8304 & 12,9186 \\
\hline 8 & +1 & +1 & +1 & 50 & 29 & 150 & 25,9256 & 37,5168 & 11,5911 \\
\hline 9 & 0 & 0 & 0 & 40 & 26 & 120 & 19,5085 & 31,0883 & 11,5798 \\
\hline 10 & 0 & 0 & 0 & 40 & 26 & 120 & 19,9702 & 32,3946 & 12,4243 \\
\hline 11 & 0 & 0 & 0 & 40 & 26 & 120 & 20,4073 & 32,7859 & 12,3786 \\
\hline
\end{tabular}


Os resultados da análise estatística aplicada aos dados experimentais de perda de peso, de perda de água e de ganho de sólidos foram determinados pelo erro puro e pelo soma dos quadrados (SS) residual.

O efeito estimado indica o quanto cada fator (variável preditora) influi nas respostas estudadas. Quanto maior for o efeito, maior será a sua influência, e um efeito positivo indica que, a variável, ao passar de um valor mínimo a um valor máximo, a resposta aumenta. Já um efeito negativo indica o contrário, ou seja, ao passar de um valor mínimo para o valor máximo, a resposta diminui (BARROS NETO et al., 2002). Isso só é válido desde que nenhuma interação apresente efeito significativo, caso contrário qualquer consideração a respeito de uma variável deve ser feita dentro do limite de outra variável.

$\mathrm{O}$ valor do coeficiente $\mathrm{P}$ está ao nível de significância da variável independente sobre a variável resposta em estudo. Normalmente é escolhido como intervalo de confiança, o valor de 95\%. Sendo assim, pode-se afirmar que, para valores de $\mathrm{P}$ inferiores 0,05 , a influência da variável preditora é considerada estatisticamente significativa (BARROS NETO et al., 2002).

Para as respostas perda de peso (PP) e perda de água (PA) os fatores significativos não foram os mesmos para erro puro e SS residual, pois para o erro puro as variáveis, temperatura, tempo e concentração foram estatisticamente significativas, enquanto para o SS residual apenas temperatura e concentração se apresentaram estatisticamente significativas, portanto a variável tempo não foi considerada estatisticamente significativa para as resposta PP e PA, a 95\% de confiança. Os parâmetros temperatura (T) e concentração (C) apresentaram efeitos positivos significativos para ambas as respostas, ou seja, um aumento em qualquer um desses fatores provoca um aumento nas respostas.

Para o ganho de sólidos, os fatores significativos não foram os mesmos para erro puro e SS residual. Pelo SS residual foram significativas as variáveis temperatura e concentração, além das interações temperatura x concentração, temperatura x tempo e tempo x concentração, enquanto que, para o erro puro, apenas as variáveis temperatura e concentração e a interação temperatura e tempo apresentaram significância estatística a $95 \%$ de confiança. No entanto, a interação tempo $\mathrm{x}$ concentração foi estatisticamente significativa a 94\%, portanto optou-se por mantê-la no modelo. O parâmetro temperatura (T) e a interação tempo e temperatura apresentaram um efeito negativo sobre a resposta ganho de sólidos. Por outro lado, o parâmetro concentração (C) e a interação tempo e concentração apresentaram um efeito positivo sobre a resposta ganho de sólidos.

Desprezando-se os fatores não significativos para a perda de peso, perda de água e ganho sólidos, realizou-se a análise de variância (ANOVA) para verificar a significância da regressão e da falta ajuste ao nível de $95 \%$ de confiança, utilizando o teste F para o planejamento estudado (Tabela 4). 
Tabela 4 - Análise de Variância (ANOVA) para perda de peso, perda de água e ganho de sólidos.

\begin{tabular}{|c|c|c|c|c|c|c|}
\hline Fonte de Variação & $\begin{array}{c}\text { Soma } \\
\text { quadrática }\end{array}$ & GL & $\begin{array}{c}\text { Média } \\
\text { Quadrática }\end{array}$ & Fcal & Ftab & $\mathbf{R}^{2}$ \\
\hline \multicolumn{7}{|c|}{ Perda de Peso } \\
\hline Regressão & 316,002 & 2 & 158,001 & 17,298 & 4,46 & 0,81 \\
\hline Resíduo & 73,069 & 8 & 9,134 & & & \\
\hline Falta de ajuste & 71,4883 & 6 & 11,915 & 15,082 & 19,33 & \\
\hline Erro Puro & 1,5805 & 2 & 0,790 & & & \\
\hline Total & 389,0709 & 10 & 38,907 & & & \\
\hline \multicolumn{7}{|c|}{ Perda de água } \\
\hline Regressão & 316,002 & 2 & 158,001 & 17,298 & 4,46 & 0,81 \\
\hline Resíduo & 73,069 & 8 & 9,134 & & & \\
\hline Falta de ajuste & 71,4883 & 6 & 11,915 & 15,082 & 19,33 & \\
\hline Erro Puro & 1,5805 & 2 & 0,790 & & & \\
\hline Total & 389,0709 & 10 & 38,907 & & & \\
\hline \multicolumn{7}{|c|}{ Ganho de sólidos } \\
\hline Regressão & 6,669 & 4 & 1,67 & 18,56 & 4,53 & 0,92 \\
\hline Resíduo & 0,558 & 6 & 0,09 & & & \\
\hline Falta de ajuste & 0,438 & 4 & 0,11 & 1,83 & 19,33 & \\
\hline Erro Puro & 0,120 & 2 & 0,06 & & & \\
\hline Total & 7,257 & 10 & 0,73 & & & \\
\hline
\end{tabular}

Segundo Barros Neto et al. (2001), uma regressão, embora significativa do ponto de vista do teste $\mathrm{F}$, pode não ser útil para realizar previsões por cobrir uma faixa de variação pequena dos fatores estudados. Box e Wetz (1973) sugeriram para que uma regressão possa ser considerada significativa, não apenas estatisticamente, mas também útil para fins preditivos, o valor de $\mathrm{F}$ calculado para a regressão deve ser, no mínimo, de quatro a cinco vezes maior que o valor de $\mathrm{F}$ tabelado. Já o $\mathrm{F}$ da falta de ajuste em relação ao erro puro, pelo contrário, deve apresentar o menor valor possível, pois um $\mathrm{F}$ alto indica que há uma grande falta de ajuste dos dados ao modelo obtido.

De acordo com a análise de variância (Tabela 4), o modelo apresentado para a perda de peso foi significativo com $95 \%$ de confiança, pois o $\mathrm{F}$ calculado foi aproximadamente quatro vezes maior que o $\mathrm{F}$ tabelado. No entanto, o modelo obtido não foi preditivo, já que o $\mathrm{F}$ da falta de ajuste foi superior ao $\mathrm{F}$ tabelado, indicando que há uma grande falta de ajuste dos dados ao modelo obtido. Assim não é possível gerar modelo para a resposta PP, uma vez que a falta de ajuste foi significativa.

A análise de variância (Tabela 4) mostrou que o modelo ajustado para a perda de água foi significativo ao nível de confiança de 95\%, e também preditivo, satisfazendo o critério sugerido por Box e Wetz (1973), visto que o F calculado foi aproximadamente 4 vezes maior que o $\mathrm{F}$ tabelado, e a falta de ajuste não foi significativa no mesmo nível de confiança. $\mathrm{O}$ coeficiente de determinação $\left(\mathrm{R}^{2}\right)$ foi de 0,81 , indicando que o modelo explicou $81 \%$ da variação dos dados observados.

A análise de variância (Tabela 4) mostrou que o modelo ajustado para a ganho de sólidos foi significativo ao nível de confiança de 95\%, satisfazendo o critério sugerido por Box e Wetz (1973), 
visto que o $\mathrm{F}$ calculado foi 4,1 vezes maior que o $\mathrm{F}$ tabelado. A falta de ajuste não foi significativa no mesmo nível de confiança. Assim além de apresentar uma regressão significativa, o modelo foi preditivo. O coeficiente de determinação $\left(\mathrm{R}^{2}\right)$ foi de 0,92 , indicando que o modelo explicou $92 \%$ da variação dos dados observados.

Os modelos codificados propostos para representar a perda de água (PA) e ganho de sólidos (GS) na desidratação osmótica de filé de peixe pirarucu, em solução de $\mathrm{NaCl}$, é o seguinte:

$$
\begin{aligned}
& P A(\%)=29,13+4,05 \mathrm{~T}+4,80 \mathrm{C} \\
& G S(\%)=11,97-0,41 \mathrm{~T}+0,46 \mathrm{C}-0,58 \mathrm{~T} . \mathrm{t}+0,34 \mathrm{C} . \mathrm{t}
\end{aligned}
$$

Obtenção da melhor condição de processo

Na Figura 1, o gráfico de PA está representado através das áreas, enquanto o GS está representado através das linhas. Observa-se uma região preta, considerada ótima para o estudo, onde se obtém uma com maior perda de água e menor ganho de sólidos.

Figura 1 - Sobreposição das superfícies de respostas e contorno de GS e PA do processo de desidratação osmótica de filés de pirarucu.

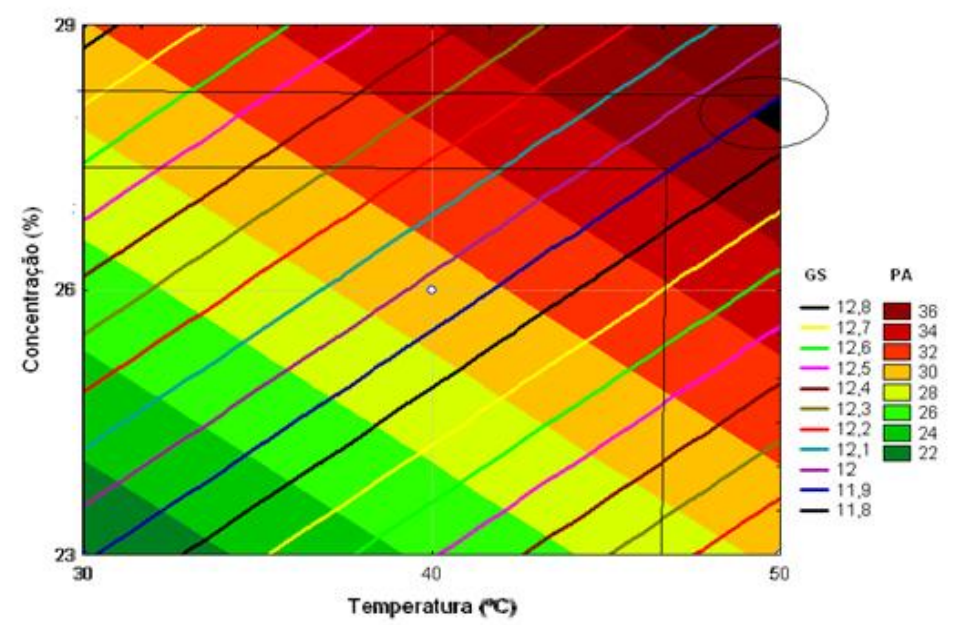

De acordo com a Figura 1, dentro da faixa de trabalho, optou-se por trabalhar com a seguinte condição ótima: temperatura de $49^{\circ} \mathrm{C}$ e concentração de $28 \%$, pois maior temperatura e maior concentração de $\mathrm{NaCl}$ potencializam uma maior desnaturação de proteínas, o que, conseqüentemente, altera mais profundamente a textura do pescado. Altas temperaturas tendem, também, a provocar oxidação de lipídeos, o que afeta a cor, o sabor e o odor do pirarucu. 


\title{
4 Conclusão
}

Após realização dos estudos sobre a desidratação osmótica de filés de pirarucu verifica-se que para as respostas perda de peso e perda de água os parâmetros temperatura e concentração apresentaram efeito significativo positivo sobre tais respostas e apresentaram regressão significativa a $95 \%$ de confiança. No entanto apenas o modelo obtido para a PA foi preditivo a 95\% de confiança. $\mathrm{O}$ parâmetro temperatura e a interação tempo $\mathrm{x}$ temperatura apresentaram efeito significativo negativo, enquanto o parâmetro concentração e a interação concentração x tempo apresentaram efeito significativo positivo para a resposta ganho de sólidos e o modelo proposto foi preditivo a $95 \%$ de confiança.

A sobreposição das superfícies de respostas das repostas PA e SG indicou que a temperatura de $49^{\circ} \mathrm{C}$ e a concentração de $28 \%$, independentemente do tempo utilizado, foi a mais viável na obtenção de maior PA e menor SG.

O produto desidratado osmoticamente na melhor condição de processo apresentou condições higiênico-sanitárias satisfatórias. Observou-se que este produto apresentou uma redução de 26,41\% de umidade em relação a matéria-prima, comprovando assim a capacidade de remoção de água do processo de desidratação osmótica.

Os estudos confirmaram a utilização da desidratação osmótica como um pré-tratamento, tendo em vista que o pirarucu desidratado osmoticamente apresentou umidade de $58,56 \%$, o que não o caracteriza como pescado seco, portanto a secagem convencional é necessária para que o produto possa atingir a umidade determinada pela legislação.

\begin{abstract}
Osmotic treatments are used mainly as a pre-treatment introduced in some conventional processes to improve the quality of the product and to reduce energy cost, as well as the formulation of new products. This study make better, with answer surface metodology, the osmotic dehydration of pirarucu meat. A central composite design $2^{3}$ was developed with independent variable temperature, concentration of $\mathrm{NaCl}$ and imersion time, with dependent variables weight loss, water loss and solid gain. The concentration of $\mathrm{NaCl}$ and the temperature were the variables that showed most influenced in the weight loss and water loss of the samples of pirarucu meat, they presented a positive effect. The temperature and the interaction time $x$ temperature presented significant negative effect, but $\mathrm{NaCl}$ concentration and the interaction of the concentration $\mathrm{NaCl} x$ time, presented significant positive effect to the answer to solid gain. The osmotic dehydration's time did not have influence in the answers in this study. The superposition of the surfaces of answers related to water loss and solid gain indicated that the temperature of $49^{\circ} \mathrm{C}$ and the concentration of $28 \%$, was the condition most viable way for obtaining better water loss and less solid gain.
\end{abstract}

Key - words: osmotic dehydration; pirarucu; planning trial. 


\section{Referências}

AOAC - ASSOCIATION OF OFFCIAL ANALYTICAL CHEMISTS. Official methods of analysis of AOAC International. Gaitheersburg: AOAC, 16 ed, 1997.

BARD. J.; IMBIRIBA, E. P. Piscicultura do pirarucu, Arapaima gigas, Belém: Embrapa - CPATU, 1986. 17p.

BARROS NETO, B.; SCARMINIO, I. S.; BRUNS, R. E. Como fazer experimentos pesquisa e desenvolvimento na ciência e na indústria. Campinas: Editora da UNICAMP, 2002.

BARROS NETO, B.; SCARMINIO, I. S.; BRUNS, R. E. Como fazer experimentos pesquisa e desenvolvimento na ciência e na indústria. Campinas: Editora da UNICAMP, 2001.

BRASIL. SECRETARIA DE VIGILÂNCIA SANITÁRIA. Portaria 451 de 19 de Setembro de 1997. Princípios Gerais para o Estabelecimento de Critérios e Padrões Microbiológicos para Alimentos. Diário Oficial da União, DF, 1997.

BRASIL. MINISTÉRIO DA AGRICULTURA, PECUÁRIA E ABASTECIMENTO. Portaria n 52 de 2000 do Ministério da Agricultura, Pecuária e Abastecimento. Regulamento Técnico de Identidade e Qualidade de Peixe Salgado e Peixe Salgado Seco. Diário Oficial União, Brasília, DF, 2000

BOX, G. E. P.; WETZ, J. Criteria for judging adequacy of estimation by an approximate response function. University of Wisconsin Technical Report, n. 9, 1973.

CARVAlho, M. A. F. Produção de defumado a frio de file de pirarucu Arapaima gigas em forno mecânico Fischer. Manaus, 1998. 133 f. Dissertação (Mestrado em Biologia de Água Doce e Pesca Interior). INPA.

GOMES, A. T.; CEREDA, M. P.; VILPOUX, O. Desidratação osmótica: uma tecnologia de baixo custo para o desenvolvimento da agricultura família. Revista Brasileira de Gestão e Desenvolvimento Regional . v. 3, n. 3, p. 212226, set./dez. 2007.

IMBIRIBA, E. P. Crescimento e produção de pirarucu, Arapaima gigas, sob diferentes densidades de estocagem em associação com búfalas leiteiras. Belém, 2001. 65 f. Dissertação (Mestrado em Ciência Animal) - Universidade Federal do Pará.

OLIVEIRA, P. R. Qualidade do pirarucu (Arapaima gigas, Schinz 1822) procedente de piscicultura, estocado em gelo, congelado e de seus produtos derivados. Manaus, 2006. 130 f. Tese (Doutorado em Ciências Biológicas). Universidade Federal do Amazonas.

ONO, E. A.; HALVERSON, M. R.; KUBTIZA, F. Pirarucu, o gigante esquecido. Revista Panorama de Aquicultura. v. 14, n. 81, p. 14-25. 2004.

OGAWA, M. Alterações da carne do pescado por processamento e estocagem. In: OGAWA, M.; MAIA, E. L. Manual de pesca (Ciência e tecnologia do pescado). São Paulo: Livraria Varela, 1999, p. 221-249.

PEREIRA-FILHO, M.; CAVEIRO, B. A. S.; ROUBACH, R.; ITUASSU, D. R.; GANDRA, A. L.; CRECÊNCIO, R.; Resultados preliminares da engorda do pirarucu (Arapaima gigas) em viveiro escavado. In: Anais do XII Simpósio Brasileiro de Aquicultura, Goiânia- GO. 24 a 29 de junho de 2002. 18p.

PIGOTT, G.; TUCKER, B. Sea effects of tecnology on nutrition, 1st edit., New York, USA: Edit. Marcel Dekker, INC, 1990.

RAOULT- WACK, A. L.; LAFONT, F.; RIOS, G.; GUILBERT, S. Osmotic dehydration of mass transfer in terms of engineering properties. In: MUJUMDAR, A. S.; ROQUES, M. A. Drying of solids. New York: Hemisphere Publishing Company. 1989, p. $487-495$.

RAOULT- WACK, A. L. Recent advances in the osmotic dehydration of food. Trends in Food Science Technology, v. 5, n. 8, p. 225-260, 1994. 
REYES, GENARA; CORZO, OTONIEL; BRACHO, NELSON. Optimización de La deshidratación osmótica de sardina mediante la metodología de superficies de respuesta. Revista Científica, Maracaibo, v. 15, n. 4, p. 377-384. 2005.

SERENO, A. M.; HUBINGER, M. D.; COMESAÑA, J. F.; CORREA, A. Prediction of water activity of osmotic solutions. Journal of Food Engineering, Holanda, v. 49, p. 103-114, 2001.

TORREGGIANI, D. Osmotic dehydration in fruit and vegetables processing. Food Research International, v. 26, n. 1, p 59-68, 1993. http://dx.doi.org/10.1016/0963-9969(93)90106-S

Submetido em 25 jan 2011, Aceito para publicação em 22 mai 2012. 